\title{
$\mathrm{CiSj}$
}

\section{USING EMBEDDED SIMULATORS FOR DEPLOYMENT-SPECIFIC ADAPTATION OF SENSOR NETWORKS}

\section{Zdravko Karakehayov}

Technical University of Sofia, Kliment Ohridski 8, Sofia-1000, Bulgaria, e-mail: zgk@tu-sofia.bg,www.tu-sofia.bg

\begin{abstract}
This paper introduces a method for deployment-specific adaptation of wireless ad hoc networks. The method utilizes sophisticated nodes which model the network signature for actual deployments and routing paths. Since the transmit power is fixed, many nodes may not save energy from communication. At the same time, all nodes save energy from calculations and the number of beacon periods may be controlled. The nodes with embedded simulators control the balance between lifetime and real-time performance. Also, some routes are modified to decline the communication range and save energy. An example shows a significant energy reduction and improved real-time performance.
\end{abstract}

Keywords: Sensor networks, low-power routing, real-time communication, network simulators.

\section{INTRODUCTION}

Ad hoc networks have a wide spectrum of military and commercial applications. Ad hoc networks are employed in situations where installing an infrastructure is too expensive, too vulnerable or the network is transient. The interaction between the nodes is based on wireless communication. Packets are forwarded in a multihop manner. Nodes have a limited radio footprint and when a node receives a packet it applies a routing algorithm to select a neighbor for forwarding.

There is a class of ad hoc networks, sensor networks, where the requirements for lifetime and size of the nodes are driven to extremes. A wireless sensor network consists of a large number of nodes that may be randomly and densely deployed. Sensor nodes are capable of sensing many types of information such as temperature, light, humidity and radiation. Sensor networks must collect data in an area of interest for months or years. Since the energy is a scarce and usually non-renewable resource, the network's functionality must be viewed from a lowpower perspective. Sensor network nodes execute three major tasks: sensing, computation and communication.

Communication energy dominates the overall energy budget. The greater than linear relationship between transmit energy and distance promises to reduce the energy cost when the radio link is partitioned. Nodes calculate the distance and tune their transmit power accordingly. Consequently, it would be beneficial to use several hops to reach a node within the transmission radius instead of a direct link. Along with available locations of nodes, a multihop optimization requires an appropriate power model. For some applications it is not necessary nodes to have real coordinates. Instead, nodes may have virtual coordinates: hop-distances to other nodes.

Moreover, some applications require the network to influence the environment via actuators. Synchronization between input and output demands real-time traffic. Real-time forwarding of packets under multihop communication scheme is a serious challenge. The multihop nature of ad hoc networks, while beneficial for energy reduction, brings the packets delivery time up. The dynamic nature of the network and the power-efficient partitioning of communication links in particular, often result in unpredictable traffic timing parameters. Enemy nodes included in a routing path may misbehave and any attempt to make the network less vulnerable requires extra energy and affects the lifetime, thus closing the loop.

Simulation has proven to be a valuable tool in cases where the alternatives are too many. Ad hoc network simulators are used to study the major parameters such as throughput, real-time and lifetime performance.

In this paper we consider heterogeneous sensor networks where sophisticated nodes execute basic simulation tasks. We call these tasks embedded 
simulators. Simulation results are employed to dynamically adapt the network to changing requirements.

\section{RELATED WORK}

Sensor network applications are described in [1, 2]. Different MAC protocols are discussed in [3-6]. Energy efficiency is the primary goal of the research. While a power saving technique, termed Span [3], dynamically splits the nodes into sleeping nodes and forwarding nodes, S-MAC, a medium access control protocol [4], establishes a low duty cycle operation in all nodes. ExOR, extremely opportunistic routing is a routing method developed to reduce the total number of transmissions taking into account the actual packet propagation [5].

In related research we proposed ALS-MAC, a medium access control protocol where contentionbased advertising slots are mapped to scheduledbased transmission slots [7]. Despite there being a plethora of sensing and MAC papers, comparatively little has been published on the companion task of actuation and real-time requirements. Sensoractuator networks are discussed in [8], [9]. The problem of obtaining virtual coordinates is addressed in [10]. Routing methods are discussed in [11]-[14].

Movement of nodes and differences of the node's lifetime outline the network dynamics. A review on routing and location updates is available in [15]. To locate nodes in space a coordinate systems is needed. The use of a single global coordinate system is not the only possibility [16]. Small sets of nodes or even single nodes may use a local coordinate system each. In case coordinates of nodes are passed across the border between the scopes of two different local coordinate systems, a coordinate transformation must be applied. Typically, a sensor network has a single base station and the interaction pattern dictates the use of a single coordinate system. Consequently, a global approach might be the better choice. Organizing a global coordinate system from local information is discussed in [17]. The global approach provides a better chance to achieve collision-free concurrent data transmissions. DTA, data transmission algebra, has been developed to generate complex transmission schedules based on collision-free concurrent data transmissions [18]. A Flexible Power Scheduling (FPS), a distributed power management protocol, is proposed in [19]. The protocol utilizes a tree-based topology in combination with an adaptive slotted communication schedule to route packets, synchronize with neighbors, and schedule the radio on/off mode.

Simulation provides an attractive method for evaluating the performance of mobile sensor networks. However, if the simulation doesn't reflect an important aspect of reality, it can't give insight into the characteristics of the network [20]. Researchers commonly model node mobility as random which is rarely the case. In related research we studied deployment-specific lifetime/real-time optimization for wireless ad hoc networks [21]. A laptop-type node collects the locations of the nodes and runs simulation tasks to optimize essential parameters for the current deployment.

\section{COMMUNICATION MODEL}

Beacon Advertise Transmit, BAT, model is a widespread MAC mechanism. Beacons are employed to synchronize internode communications. A beacon period, $T_{B}$, includes two major sections. The period begins with a traffic indication window, $\mathrm{T}_{\mathrm{A}}$. During $\mathrm{T}_{\mathrm{A}}$ all nodes are listening and pending packets are advertised. The nodes addressed till the end of $\mathrm{T}_{\mathrm{A}}$ send acknowledgements and receive data packets. Data transmissions are followed by acknowledgement frames to confirm successful reception. Fig. 1 illustrates a beacon period.

The BAT model has five parameters: $\mathrm{T}_{\mathrm{A}}, \mathrm{T}_{\mathrm{B}}$, a data packet length in bits, $\mathrm{p}$, a control packet length in bits, q, and a bit rate, B.

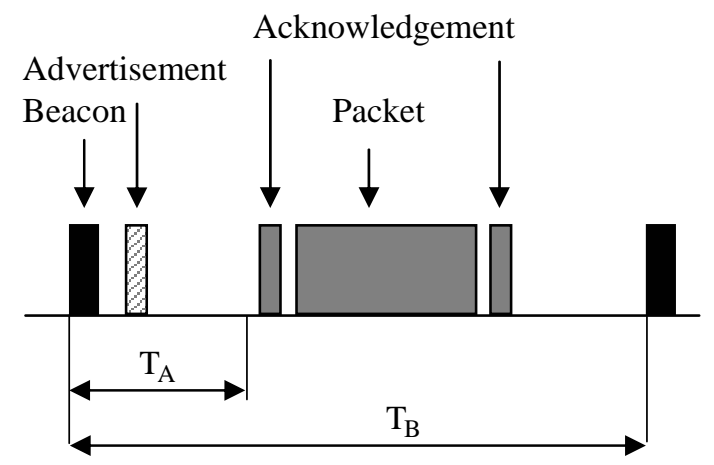

Fig. 1 - Beacon period

\section{ENERGY MODEL}

The energy used to send a bit over a distance $d$ via radio communication may be written as

$$
\mathrm{E}=a d^{n}+b
$$

where $a$ - proportionality constant [22]. The radio parameter $n$ is a path loss exponent that describes the rate at which the transmitted power decays with increasing distance. Typically, $n$ is between 2 and 4 . The $b$ constant is associated with specific receivers, CPUs and computational algorithms.

The energy model is relevant for nodes located near to the ground. 


\section{ROUTING ALGORITHMS}

Routing algorithms can be based on two major approaches: topology-based and position-based routing. The topology-based algorithms can be further split into table-driven and demand-driven. The main idea behind the table-driven routing protocols is to create a clear picture of all available routes from each node to every other node in the network. In contrast to the table-driven protocols, the demand-driven algorithms create routes via route discovery procedures only when a necessity arises.

Position-based routing algorithms utilize the physical positions of the participating nodes. Positionbased or geographic routing does not require each node to have the locations of all other nodes. Each node keeps track of the coordinates of its neighbors and their neighbors. A greedy routing algorithm based on geographic distance selects the closest to the destination neighbor for the next hop [11, 14].

In a two-hop distance approach, each node maintains a table of all immediate neighbors as well as each neighbor's neighbors. The number of hops taken into account determines the vulnerability of the routing in case of topology holes. However, considering more hops will require longer execution times. Fig. 2 shows how the transition from a single hop to two hops brings the execution time up. The code has been written in $\mathrm{C}$ and compiled for two CPUs: 8051 and Atmel AVR [14].

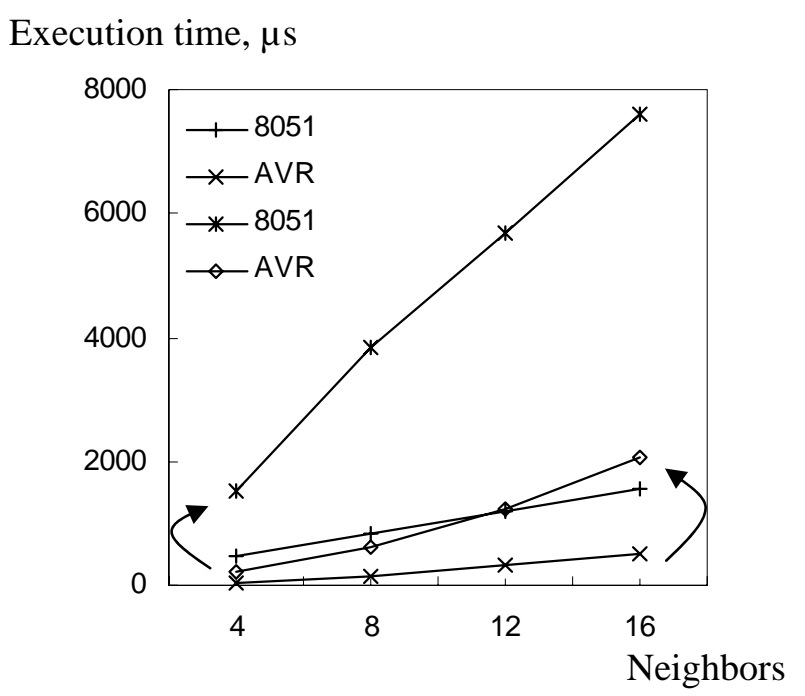

Fig. 2 - Execution time to select the next relay

\section{EMBEDDED SIMULATORS}

The network topology depends on the locations of the nodes and the communication range. Fig. 3 shows a network topology for a communication range of 150 $\mathrm{m}$. Even though there is a single isolated node, 33, uncovered areas indicate connectivity problems. Fig. 4 shows the network topology for a communication range of $200 \mathrm{~m}$. The number of neighbors is increased. Again there are some areas which are impossible to pass.

Fig. 5 shows a typical communication pattern routing packets to a base station. The base station is a sophisticated node capable of running simulation tasks. Fig. 6 shows the throughput, the number of beacon periods and the energy for different communication ranges. An embedded simulator runs a simulation task to select a suitable communication range for all nodes. The selected communication range is broadcast and adopted from the network. Also, the simulator can be used to correct the routing algorithm for certain hops. The problem is that routing algorithms typically consider one or two hops. Using global view of the network allows not only to avoid dead ends, but also to work at low communication ranges.

The simulation procedure begins with a certain communication range which is gradually increased to reach a reasonable throughput. Then the sources of the missing packets are checked for connectivity to nodes that have already been linked to the tree. In case of connectivity, the simulator instructs nodes to take specific routes instead of following the algorithm. For the example shown in Fig. 5 nodes 5, 11, 21 and 28 are instructed to forward packets to node 15. Fig. 7 shows related simulations. Based on the throughput, the communication range is declined from $220 \mathrm{~m}$ to $150 \mathrm{~m}$. The energy is decreased from $32.5 \mathrm{~mJ}$ to $5.8 \mathrm{~mJ}$. The packets delivery time is declined as well. The simulation assumes $\mathrm{T}_{\mathrm{A}}=1 \mathrm{~ms}$, $\mathrm{T}_{\mathrm{B}}=8 \mathrm{~ms}$ and packets lengths of 128 bits. The rate would be $250 \mathrm{Kbps}$. In case of variable transmit power the energy used for communication would be 25 mJ. Using a single communication range simplifies simulations which is important for embedded in the network tools.

\section{CONCLUSION}

In this paper we have presented a method for deployment-specific adaptation of wireless ad hoc networks. The method utilizes embedded simulators to model the network signature for actual deployments and routing paths. Since the transmit power is fixed, many nodes may not save energy from communication. At the same time, all nodes save energy from calculations and the number of beacon periods may be reduced. The sophisticated nodes that execute simulation tasks control the balance between lifetime and real-time performance via optimal communication ranges. Also, the simulation results are used to instruct some nodes to modify routing paths. In this way the required throughput is achieved with smaller communication ranges. An example shows a significant energy reduction and improved real-time performance. 


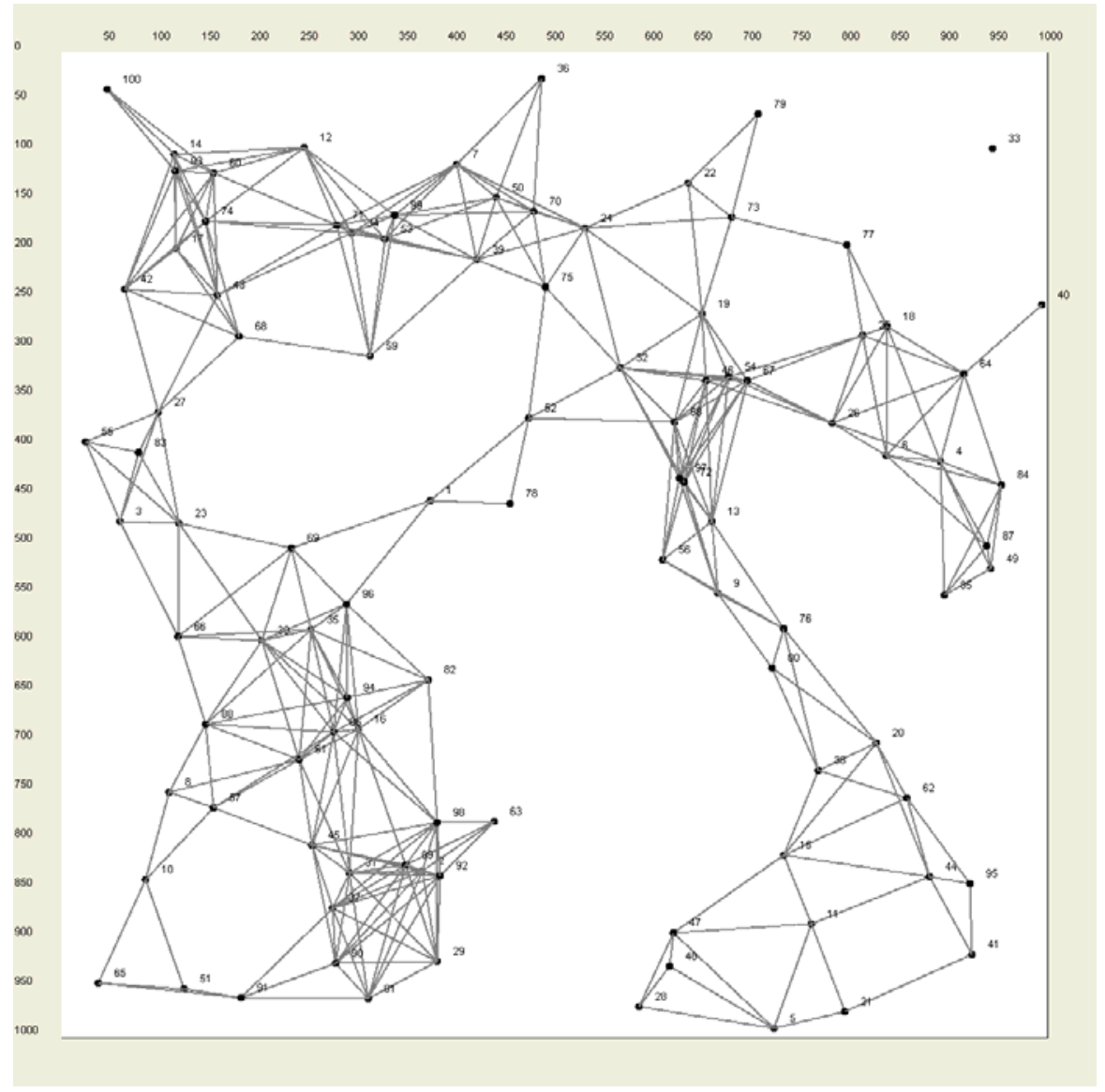

Fig. 3 - Network topology for $R=150 \mathrm{~m}$

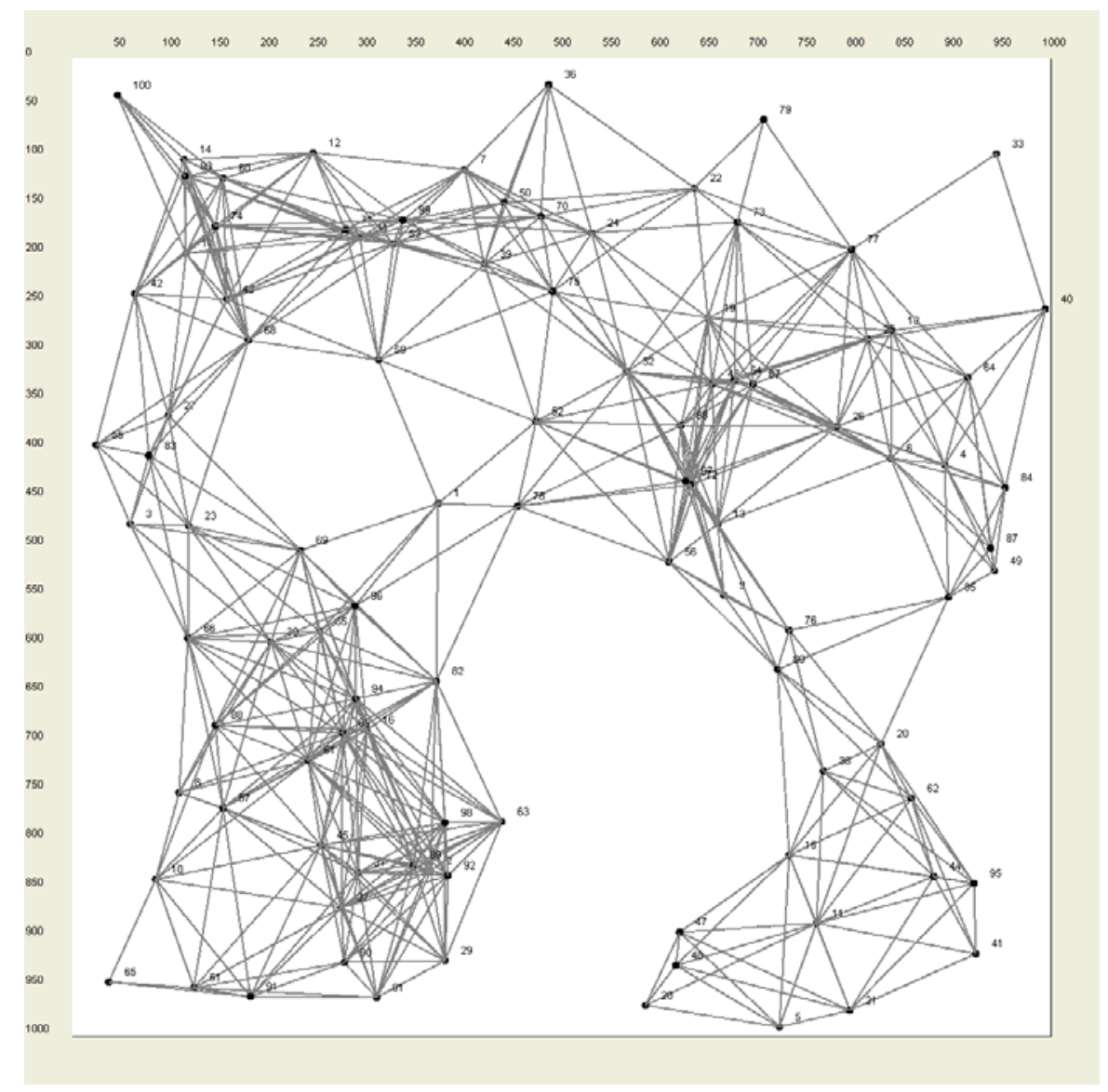

Fig. 4 - Network topology for $R=200 \mathrm{~m}$ 


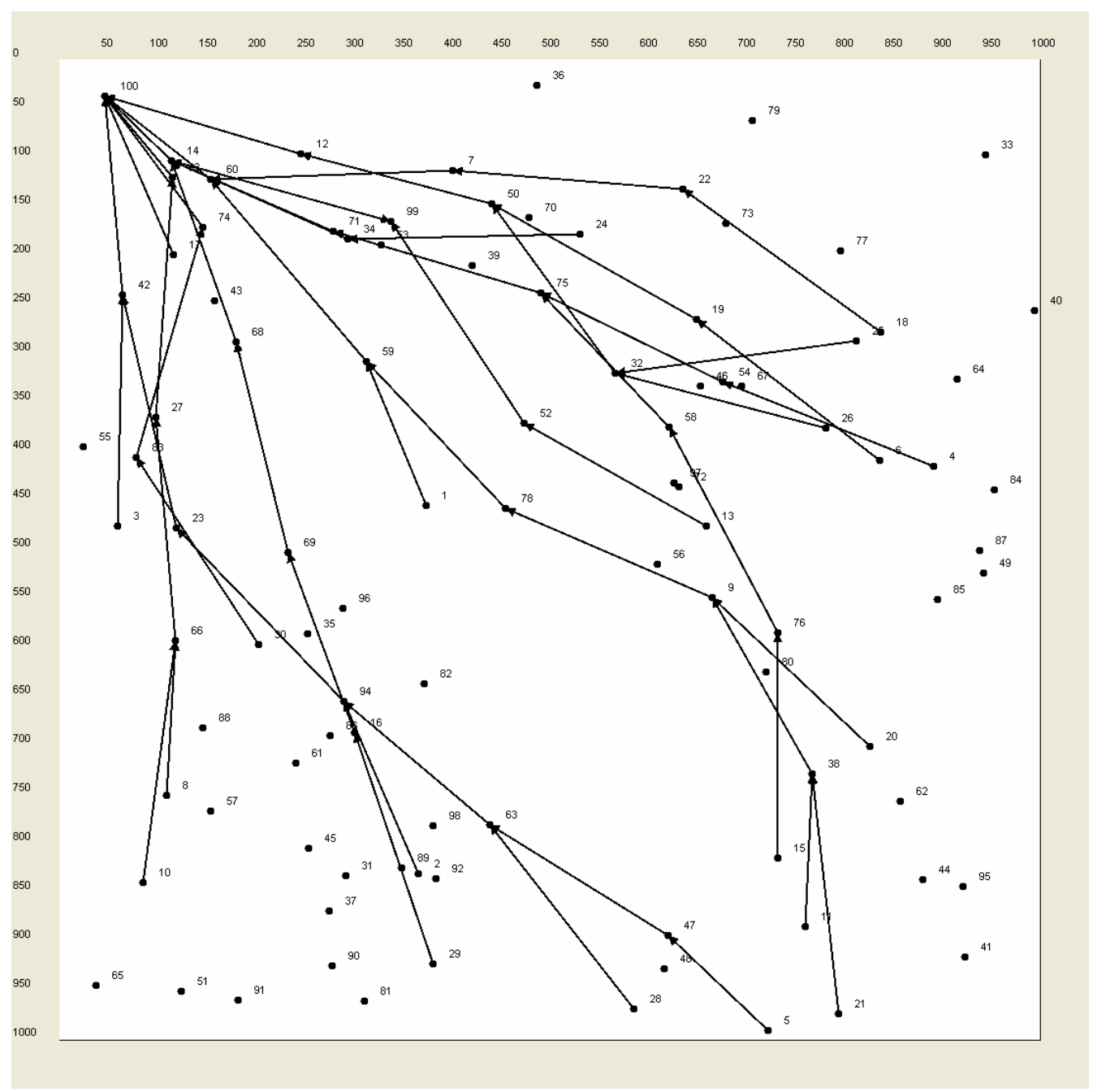

Fig. 5 - Routing packets to a base station

Throughput

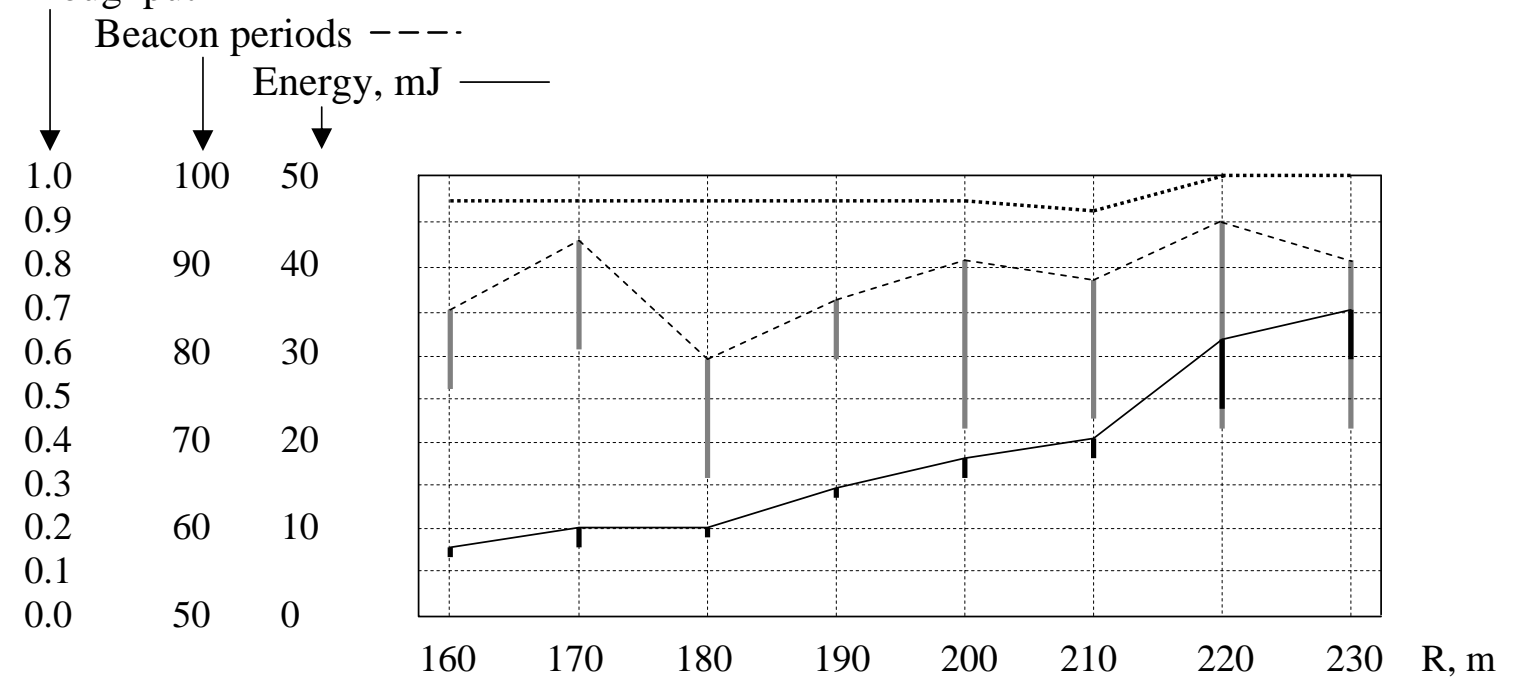

Fig. 6 - Simulation results 


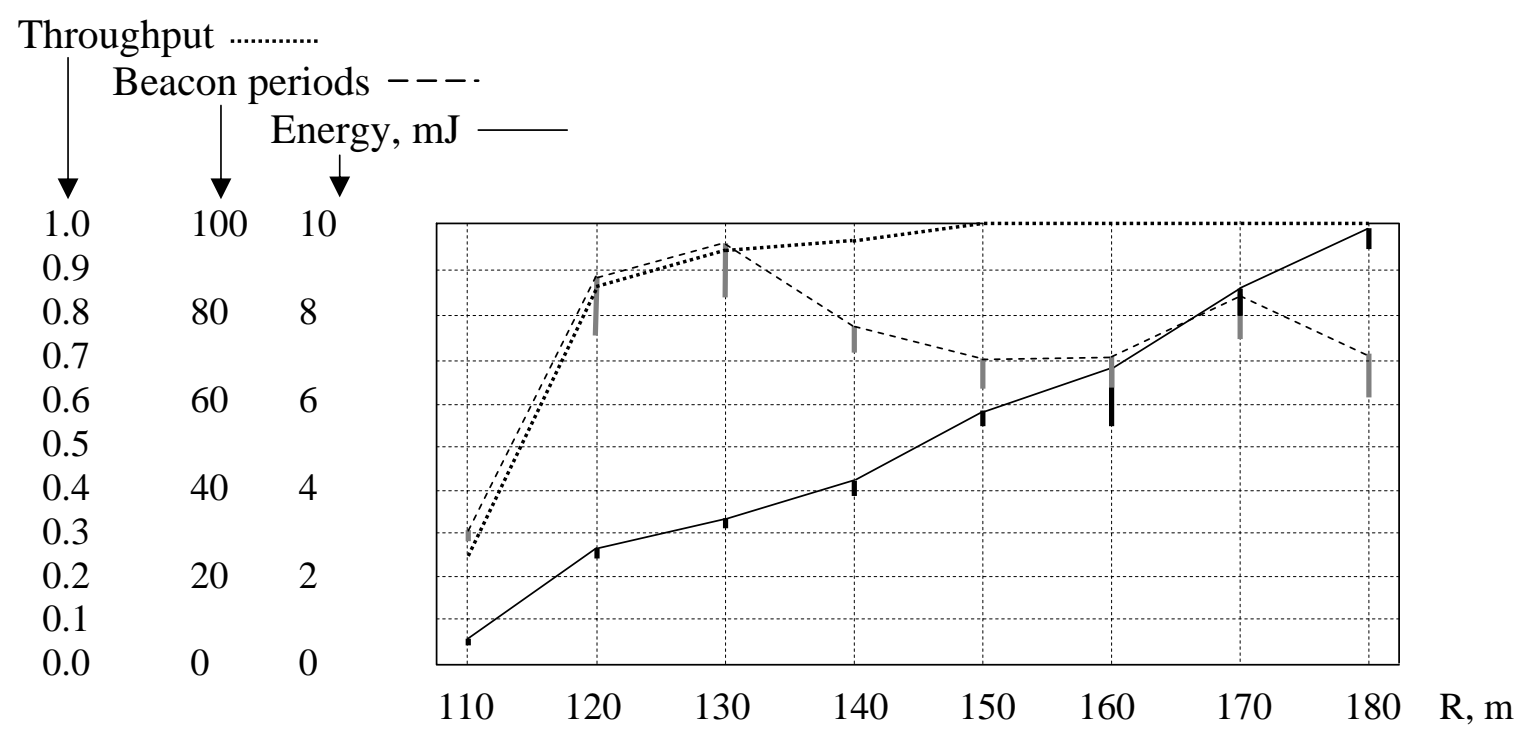

Fig. 7 - Simulation results for the modified tree

\section{ACKNOWLEDGMENT}

This work was supported in part by SE Europe Programme under project SEE AF/A/219/1.1/X I3E. Ivan Radev and Veneta Dencheva have written the code for the network simulator.

\section{REFERENCES}

[1] Q. Wang, H. Hassanein, K. Xu. A practical perspective on wireless sensor networks. Handbook of Sensor Networks: Compact Wireless and Wired Sensing Systems, edited by Mohammad Ilyas and Imad Mahgoub, CRC Press LLC, 2005, pp. 9-1 - 9-28.

[2] D. Puccinelli, M. Haenggi. Wireless sensor networks: applications and challenges of ubiquitous sensing. IEEE Circuits and Systems Magazine, third quarter 2005, pp. 19-29, .

[3] B. Chen, K. Jamieson, H. Balakrishnan, R. Morris. Span: An energy-efficient coordination algorithm for topology maintenance in ad hoc wireless networks. ACM Wireless Networks J., 2002, vol. 8, no. 5, pp. 481-494.

[4] W. Ye, J. Heidemann, D. Estrin. Medium access control with coordinated adaptive sleeping for wireless sensor networks. IEEE/ACM Transactions on Networking, vol. 12, no. 3, June 2004, pp. 493-506.

[5] S. Biswas, R. Morris. Opportunistic routing in multi-hop wireless networks. ACM SIGCOMM Computer Communication Review, vol. 34, Issue 1, Jan. 2004, pp. 69-74.

[6] D. Dewasurendra, A. Mishra. Design challenges in energy-efficient medium access control for wireless sensor networks. Handbook of Sensor Networks: Compact Wireless and
Wired Sensing Systems, M. Ilyas and I. Mahgoub, Eds., CRC Press LLC, 2005, pp. 281-28-25.

[7] Z. Karakehayov, N. Andersen. Energy-efficient medium access for data intensive wireless sensor networks. Proc. Int. Workshop on Data Intensive Sensor Networks, IEEE ISBN: 14244-1241-2, 8th Int. Conference on Mobile Data Management, Mannheim, Germany, May 2007, pp. 116-120.

[8] I. F. Akyildiz, I. H. Kasimoglu. Wireless sensor and actor networks: research challenges. Ad Hoc Networks, 2, 2004, pp. 351-367.

[9] Z. Karakehayov. Low-power communication for wireless sensor-actuator networks. Proc. of the Fifth IASTED Int. Conference on Communication Systems and Networks, Palma de Mallorca, Spain, ACTA Press, Aug. 2006, pp. 1-6.

[10] T. Moscibroda, R. O'Dell, M. Wattenhofer, R. Wattenhofer. Virtual coordinates for ad hoc and sensor networks. ACM Joint Workshop on Foundations of Mobile Computing (DIALMPOMC), Philadelphia, USA, October 2004.

[11] I. Stojmenovic. Position-based routing in ad hoc networks. IEEE Communications Magazine, July, 2002, pp. 128-134.

[12] G. Vakulya, G. Simon. Percolation driven flooding for energy efficient routing in dense sensor networks. Journal of Telecommunications and Information Technology, 2009. Editors Pawel Gburzynski, University of Alberta and Jozef Wozniak, Gdansk University of Technology, pp. 5-12.

[13] Z. Karakehayov. Low-power design for Smart Dust networks. Handbook of Sensor Networks: Compact Wireless and Wired Sensing Systems, 
M. Ilyas and I. Mahgoub, Eds., CRC Press LLC, 2005, pp. 37-1-37-12.

[14] Z. Karakehayov, Z. Monov. Target-aware timing modelling for wireless ad-hoc networks. Proceedings International Scientific Conference Computer Science'2006, October, 2006, Istanbul, pp. 54-59.

[15] I. Stojmenovic. Location updates for efficient routing in ad hoc wireless networks. Handbook of Wireless Networks and Mobile Computing, Wiley, 2002, pp. 451-471.

[16] K. Romer, F. Mattern. Towards a unified view on space and time in sensor networks. Computer Communications, 28 (2005), pp. 1484-1497.

[17] R. Nagpal, H. Shrobe, J. Bachrach. Organizing a global coordinate system from local information on an ad hoc sensor network. Proceedings $2^{\text {nd }}$ Int. Workshop on Information Processing in Sensor Network, 2003, http:/groups.csail.mit.edu/mac/projects/amorph ous/papers/ipsn-2003-v5.pdf.

[18] V. Zadorozhny, D. Sharma, P. Krishnmurthy, A. Labrinidis. Tuning query performance in mobile sensor databases. Proc. 6th Int. conference on Mobile data management, Ayia Napa, Cyprus, 2005, pp. 247-251.

[19] B. Hohlt, L. Doherty, E. Brewer. Flexible power scheduling for sensor networks. Proceedings $3^{\text {rd }}$ Int. Symposium on Information Processing in Sensor Networks, 2004, pp. 205214.

[20] T. R. Andel, A. Yasinsac. On the credibility of Manet simulations. IEEE Computer, July 2006, pp. 48-54.
[21] Z. Karakehayov. Deployment-specific lifetime / real-time optimization for wireless ad hoc networks. IEEE Int. Workshop on Intelligent Data Acquisition and Advanced Computing Systems, Rende (Cosenza) Italy, 21-23 September, 2009, pp. 661-664.

[22] J. M. Rabaey, M. J. Ammer, J. L. Silva, D. Patel, S. Roundy. PicoRadio supports ad hoc ultra-low power wireless networking. IEEE Computer, vol. 33, Jul. 2000, pp. 42-48.

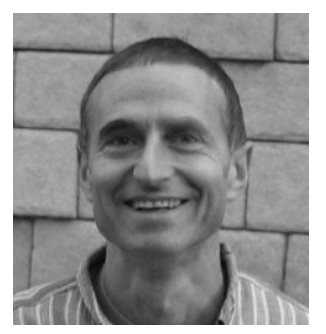

Zdravko Karakehayov is an Associate Professor in the Department of Computer Systems at the Technical University of Sofia, Bulgaria. Formerly he was with the Technical University of Denmark, Lyngby and the

University of Southern Denmark, MCI-CPD, Sønderborg. Dr. Karakehayov received the Ph.D. degree in computer science from Technical University of Sofia, Bulgaria.

$\mathrm{He}$ is a senior member of the IEEE Computer Society. Dr. Karakehayov currently chairs the Computer Chapter, IEEE Bulgarian section. He coauthored five books in the field of embedded systems and holds eight patents.

His research field includes low-power design for embedded systems, low-power and secure routing for wireless sensor networks. Dr. Karakehayov served as a reviewer for the Journal Transactions on Embedded Computing Systems and several international conferences. 